\title{
EXPERIMENTAL STUDIES OF INTENT INFORMATION ON COCKPIT TRAFFIC DISPLAYS
}

\author{
Richard Barhydt \\ R. John Hansman, Jr. \\ Aeronautical Systems Laboratory \\ Massachusetts Institute of Technology \\ Cambridge, MA 02139
}

\begin{abstract}
Intent information provides knowledge of another aircraft's current and future trajectory states. Prototype traffic display's were designed for four different levels of intent: No Intent, Rate, Commanded State, and FMS-Path. The TCAS Display was used as a baseline and represents the No Intent Level. The Rate, Commanded State, and FMS-Path Displays show increasing levels of intent information using TCAS-like symbology in addition to incorporating a conflict probe and profile view display. An experiment was run on the MIT Part Task Flight Simulator in which eight airline pilots flew five traffic scenarios with each of the four displays. Results show that pilots had fewer separation violations and maneuvered earlier with the three intent displays. Separation violations were reduced when pilots maneuvered earlier. A second experiment was run to compare performance between displaying intent information directly and incorporating it into a conflict probe. A different set of eight airline pilots flew four traffic scenarios with the TCAS and Commanded State Displays with and without the conflict probe. Conflict probes with two minute and long range look-ahead times were tested. Displaying conflict bands or showing intent information directly both led to fewer separation violations nce maneuvers than the base TCAS Display. Performance was similar between the two minute and long range look-ahead conflict probes. Pilots preferred all intent displays over the TCAS Display.
\end{abstract}

\section{INTRODUCTION}

In the early 1980's, significant work was done toward the development of a Cockpit Display of Traffic Information (CDTI). Many experiments showed that predictor information added to intruder airplane symbols aided pilots in determining the future position of those aircraft (Palmer, Jago, Baty, \& O’Connor, 1980 and Palmer, 1983). However, the traffic display used in conjunction with the Traffic Alert and Collision Avoidance System (TCAS) in today's commercial aircraft shows only the current position and altitude of intruder aircraft.

With the evolution toward a more unstructured air traffic management system underway, pilots may eventually incur more responsibility for maintaining separation from other traffic. To make informed decisions about potential conflicts, pilots will likely need more information about an intruder aircraft's current and future trajectory states. This information, known as "aircraft intent" exists at many different levels, with higher levels representing a more complete knowledge about the other aircraft's trajectory. It is presumed that this information could be exchanged between aircraft via an air to air datalink. Four different levels of intent were evaluated in an exploratory experiment: TCAS, Rate, Commanded State, and FMS-Path.

\section{EXPERIMENT 1}

Objective

An experiment was conducted to determine whether intent information could improve pilots' abilities to recognize and resolve traffic conflicts. To evaluate pilots' ability to use the information, prototype displays corresponding to each level of intent were developed. Subject pilots flew five different traffic scenarios with each of the four displays. Scenarios were designed to represent a variety of common traffic situations. The scenarios tested included an intruder overtake, intruder trajectory change, ownship overtake, long range conflict, and dual conflict. An intruder level-off safely above the subject pilot's aircraft was also considered. Scenarios included six background aircraft in addition to the conflict causing aircraft.

\section{Prototype Displays}

It was assumed that the appropriate level of intent information would be available for all intruder aircraft. The information provided on the prototype displays is shown in Table 1. Traffic information was superimposed onto a plan view Electronic Horizontal Situation Indicator (EHSI). The Rate, Commanded State, and FMS-Path Displays also incorporate a conflict probe and profile view display. The conflict probe uses current state information for the ownship and intruder aircraft to predict where the spacing will be within specified limits. On the FMS-Path Display, conflicts are also predicted based on the intruder's Lateral (LNAV) and Vertical (VNAV) Navigation paths. Sample Commanded State and FMS-Path Displays are shown in Figure 1. 
Table 1: Information Provided on Prototype Displays

\begin{tabular}{cll} 
Display & \multicolumn{1}{c}{ Information Shown } & \multicolumn{1}{c}{ Display Features } \\
\hline TCAS & $\begin{array}{l}\text { Current Position, Altitude, Altitutde Rate, } \\
\text { and Aircraft ID }\end{array}$ & Plan View Display \\
Rate & TCAS information and Current Heading & Conflict Probe, Plan and Profile View Displays \\
$\begin{array}{c}\text { Commanded } \\
\text { State }\end{array}$ & $\begin{array}{l}\text { TCAS information, Current Heading, } \\
\text { Commanded Heading, and Commanded Altitude }\end{array}$ & Conflict Probe, Plan and Profile View Displays \\
FMS-Path & TCAS information, LNAV and VNAV Paths & Conflict Probe, Plan and Profile View Displays \\
\hline
\end{tabular}
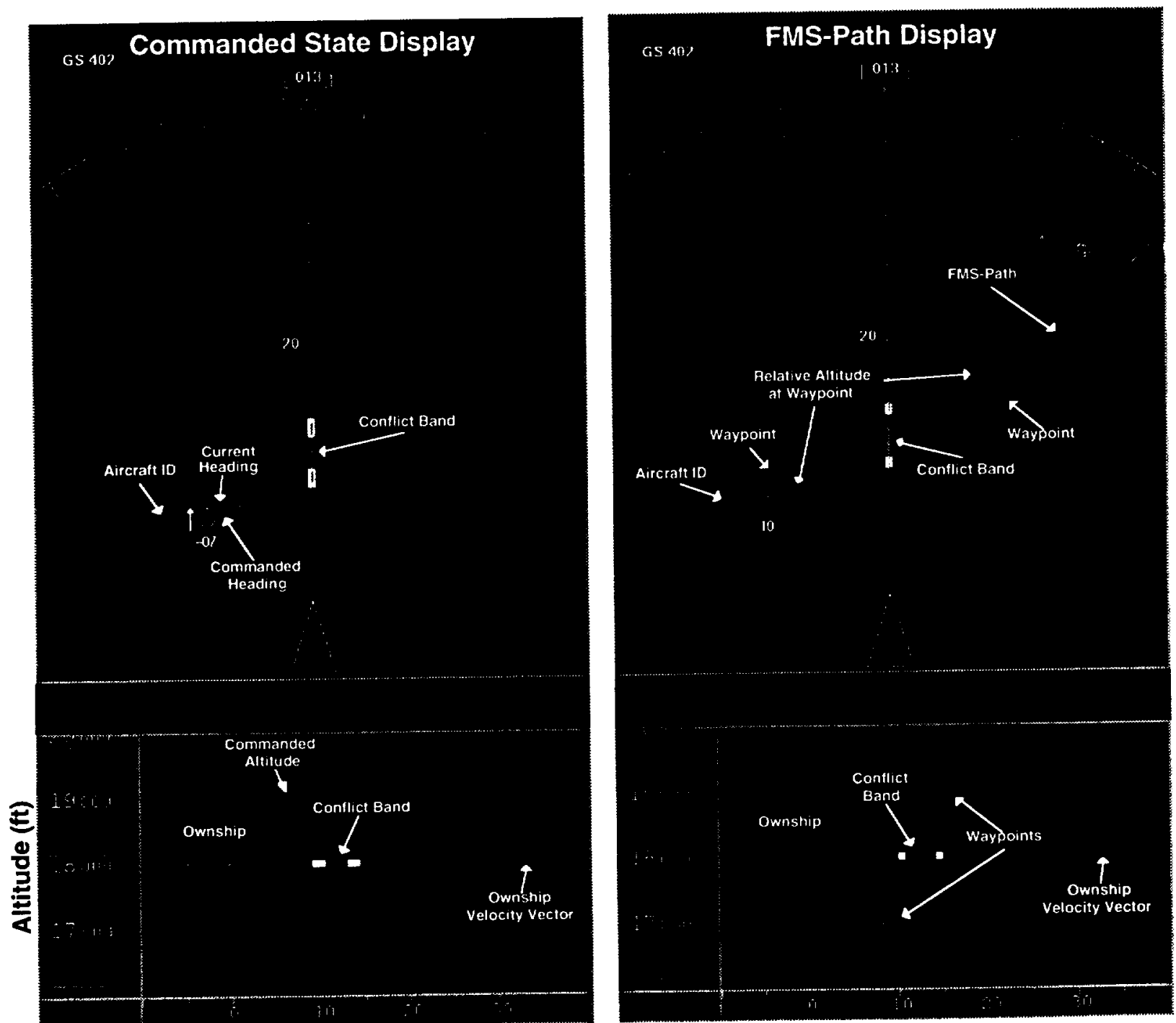

Distance Along Flight Path (nm)

Figure 1: Commanded State and FMS-Path Displays

The Commanded State Display shows the current and commanded heading referenced to ground coordinates. As part of the conflict probe, a conflict band is drawn along the ownship flight path if the projected spacing is less than $2 \mathrm{~nm}$. laterally and $500 \mathrm{ft}$. vertically (red) or $3 \mathrm{~nm}$. laterally and $700 \mathrm{ft}$. vertically (yellow). Conflict prediction is based only the current velocity of each aircraft. The aircraft ID, drawn next to the traffic symbol, changes color to correspond to the level of threat. The profile view display shows a projection of the traffic situation onto the ownship velocity vector. Distance is plotted ahead of the ownship and at the absolute altitude. 
The FMS-Path Display shows the LNAV and VNAV paths, including future waypoints and crossing altitudes. Waypoint crossing altitudes are with respect to the ownship's predicted altitude when the intruder arrives at the waypoint. The conflict probe considers both FMS-Path and current state information when predicting conflicts.

The Rate Display does not show the commanded heading or commanded altitude, but is otherwise identical to the Commanded State Display. In addition, an up or down arrow is drawn from the traffic symbol on the profile view display if the intruder is climbing or descending at least $500 \mathrm{fpm}$. The TCAS Display shows the standard information including the current position, altitude, and altitude rate arrow on a plan view display. The Aircraft ID is also provided.

Methods

Apparatus. The experiment was conducted on the MIT Part Task Flight Simulator. The simulator consists of a Mode Control Panel (MCP), Throttle Quadrant, Control Display Unit (CDU), and a control side-stick. Displays were shown on a Silicon Graphics Workstation. Pilots were provided with a B-747-400 Primary Flight Display, Flap and Gear Indicators, and the EHSI/Traffic Display. The display range could be adjusted with keyboard buttons. In an effort to reduce display clutter, intent information was only shown for aircraft projected to cause a conflict. Pilots could choose to show intent information for non-conflict aircraft by clicking on the aircraft symbol with the computer mouse.

Procedure. The primary objective of each scenario was to maintain a minimum separation of $2 \mathrm{~nm}$. laterally or $500 \mathrm{ft}$. vertically from all other traffic. Pilots were responsible for providing their own traffic separation, without help from Air Traffic Control, and were free to maneuver in any manner, at any time. Unless maneuvering to avoid a conflict, pilots were to fly direct to a VOR at a constant altitude of $18,000 \mathrm{ft}$. They were instructed to turn back to the VOR and return to $18,000 \mathrm{ft}$. as soon as possible after resolving a conflict. After the experiment, subject pilots filled out a questionnaire, asking them to rate the displays and the various elements of intent information.

Performance Metrics and Data Analysis. The primary objective performance metrics were the occurrence of separation violations (pilot was unable to maintain the minimum required spacing) and the time an avoidance maneuver was conducted. Paired t-tests were used to test for significance between the means. Pilot rankings of the displays were analyzed with the Analytic Hierarchy Process (AHP) (Yang \& Hansman, 1995). AHP uses paired comparisons of all possible display combinations to produce overall rankings for each display that sum to one.

Results

Subjects. Eight current commercial airline pilots from major US carriers served as volunteer test subjects in the experiment. They all had experience on glass cockpit and TCAS equipped aircraft. Their experience ranged from 5,000 to 24,000 hours with a mean of 10,875 hours and their age ranged from 39 to 53 with a mean of 46 .

Objective Data. The percentage of conflicts resulting in a separation violation and the maneuver time are plotted vs. display type in Figure 2. There were six possible conflicts per pilot per display, leading to a total of 48 conflicts per display. Error bars represent one standard error. The conflicts were designed to be difficult, so that differences in performance between displays could be detected.
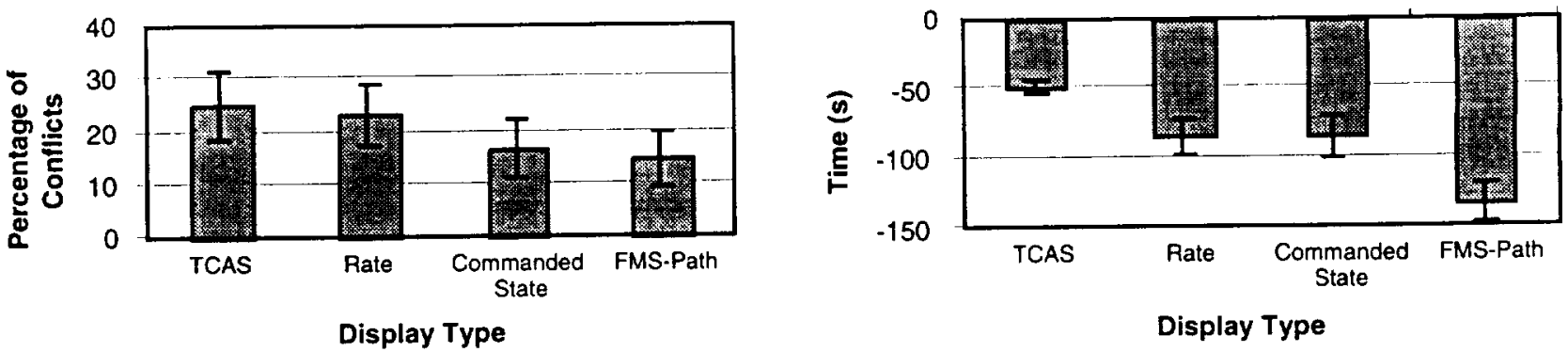

Figure 2: Separation Violation Percentage and Time Before Separation Violation to Conduct Avoidance Maneuver

There appears to be a trend toward fewer separation violations with higher levels of intent information, however differences are not statistically significant when considered over all scenarios. Pilots maneuvered earliest with the FMS-Path Display, followed by the Rate and Commanded State Displays, and latest with the TCAS Display. These differences are statistically significant $(p<0.01)$. Since the FMS-Path Display considered both the FMS-Path and current state information when predicting conflicts, its conflict band appeared an average of $72 \mathrm{~s}$. earlier than the conflict band on the Rate and Commanded State Displays. As shown in Figure 3, maneuvering earlier generally led to fewer separation violations. 


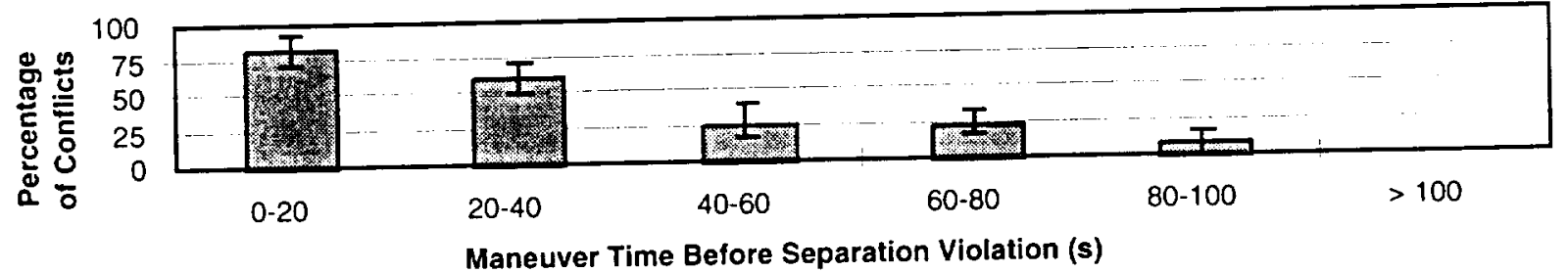

Figure 3: Separation Violation Percentage vs. Maneuver Time

Subjective Data. The overall rankings for pilot display preference are shown in Figure 4.

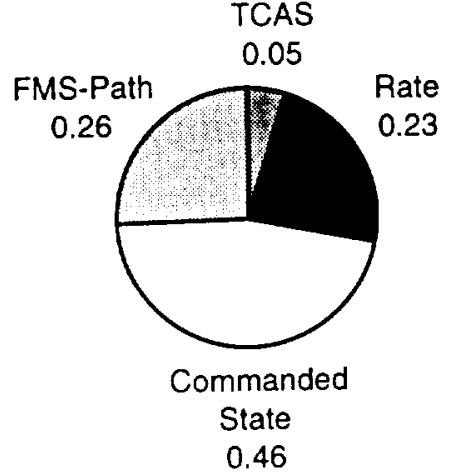

Pilots greatly preferred all of the intent displays to the TCAS Display. The Commanded State Display had the highest overall ranking, being preferred by pilots twice as much as the Rate and FMS-Path Displays and nine times as much as the TCAS Display.

In addition to rating the displays, pilots were asked to indicate which displays were too cluttered.

Sixty-three percent of the pilots found the FMS-Path Display to be too cluttered, as compared to $13 \%$ for the Commanded State Display and none for the TCAS or Rate Displays.

Figure 4: Pilot Display Preference

Discussion. Results indicate that there is a benefit to some level of intent information above the basic TCAS. There were fewer separation violations with the intent displays and they were preferred by the pilots. Showing only the next target state appears to be a more viable alternative than displaying the entire FMS-Path. Performance was very similar between the FMS-Path and the Commanded State Displays. Also, an actual LNAV/VNAV path would be difficult to show, due to uncertainties in predicting the vertical path and differences in architecture between flight management systems. In addition, over sixty percent of the pilots felt the FMS-Path Display was too cluttered.

Encouraging pilots to maneuver earlier appears to be an important asset for a display. Few separation violations occurred when pilots maneuvered several minutes before a conflict. The significant difference in maneuver times between the TCAS and Rate Displays could be attributed to any of the three additional features on the Rate Display: display of rate information, conflict bands, or the profile view display. Earlier maneuver times with the FMS-Path Display when compared to the Commanded State Display are likely due to the earlier appearance of the conflict band, since the maneuver times were similar on scenarios where the conflict band appeared at the same time.

\section{EXPERIMENT 2}

Objective

A second experiment was conducted to explore the individual and collective effect of different levels of displayed and applied intent information. The TCAS and Commanded State Levels were tested as two different ways to display intent information to the pilot. The Commanded State Level was chosen because it performed as well as the other intent levels on Experiment 1, was preferred by pilots, and would be easier to implement on a real airplane than the FMS-Path Level. Intent information can also be incorporated into the display through the use of a conflict probe. To compare the performance of short and long range alerting, cases were run with conflict bands using two minute and long range lookahead times. The look-ahead time of the long range conflict probe was longer than any of the scenarios used during the experiment. Cases without the conflict probe were also run. These three conflict band options were tested with the TCAS and Commanded State Displays, leading to six display conditions. All cases were run with four different traffic scenarios: short range conflict, intruder maneuvering during conflict, long range conflict, and intruder overtake.

\section{Methods}

The experimental apparatus and procedure were the same as in Experiment 1. Displays were changed to include only a plan view, so that performance could be compared with the plan/profile view displays of Experiment 1. Also, pilots were unable to de-select intent information for non-conflicting traffic, as they had in Experiment 1. The post experiment questionnaire asked pilots to rate displays with and without conflict bands and displayed intent information and to give 
their opinion on the proper range and look-ahead time for the conflict probe. Paired t-tests were again used to test for significance between means. Error bars on all plots represent one standard error.

Results

Subjects. Eight commercial airline pilots were used as test subjects for the second experiment. Six were active and two had retired within the preceding four months. All had experience on glass cockpit aircraft. Their flight experience ranged between 8,300 and 28,000 hours with a mean of 16,225 hours. Their ages ranged from 35 to 60 with a mean of 49.

Objective. The percentage of separation violations with each display option is shown in Figure 5. Each of the six display options of displayed and applied intent information had four possible conflicts per pilot. There were eight pilots, leading to a total of 32 conflicts per display option.

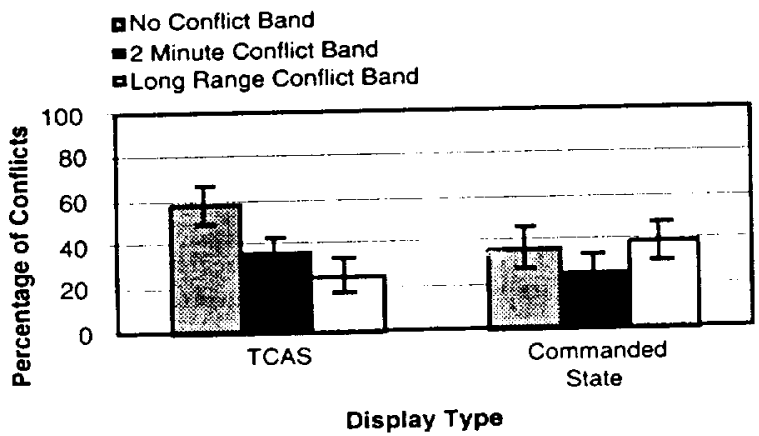

Again, the conflicts were designed to be difficult. The results show that the addition of either conflict bands or intent information to the base TCAS Display resulted in fewer separation violations. Every display option performed significantly better than the TCAS Display alone $(p<0.05)$. The differences between the two minute and long range conflict bands for each display type are not statistically significant.

Figure 5: Separation Violation Percentage vs. Display Option

The maneuver time before separation violation and after a conflict band appeared are plotted vs. display option in Figure 6. Showing conflict bands or intent information directly caused pilots to maneuver significantly earlier than the base TCAS Display $(p<0.05$ ). With the two minute conflict band, pilots maneuvered $42 \mathrm{~s}$. after the band appeared when using the TCAS Display and only $14 \mathrm{~s}$. after with the Commanded State Display. This difference is significant $(p<0.01)$. Pilots waited much longer to maneuver for conflict bands using a long range look-ahead $(p<0.01)$.

- No Conflict Band

- 2 Minute Conflict Band

- Long Range Conflict Band

TCAS

Display Type

Commanded

State

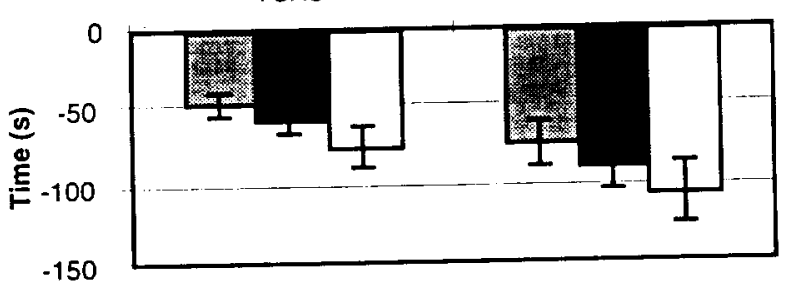

- 2 Minute Conflict Band Display Type

- Long Range Conflict Band

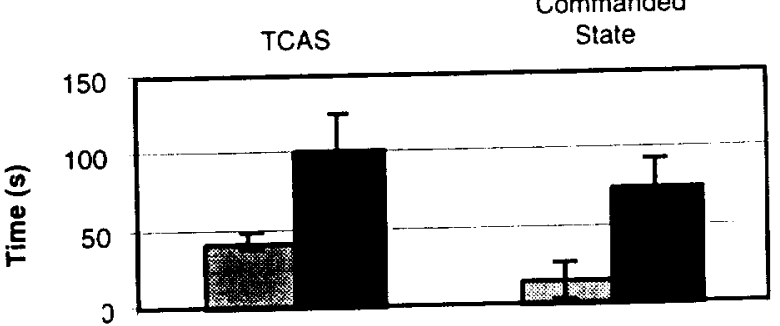

Figure 6: Maneuver Time Before Separation Violation (left) and After Conflict Band Appeared (right)

As in Experiment 1, pilots were more able to avoid separation violations if they maneuvered earlier. A histogram of separation violation percentage vs. maneuver time is shown in Figure 7. The most significant improvement occurred for maneuvers within two minutes of the conflict.

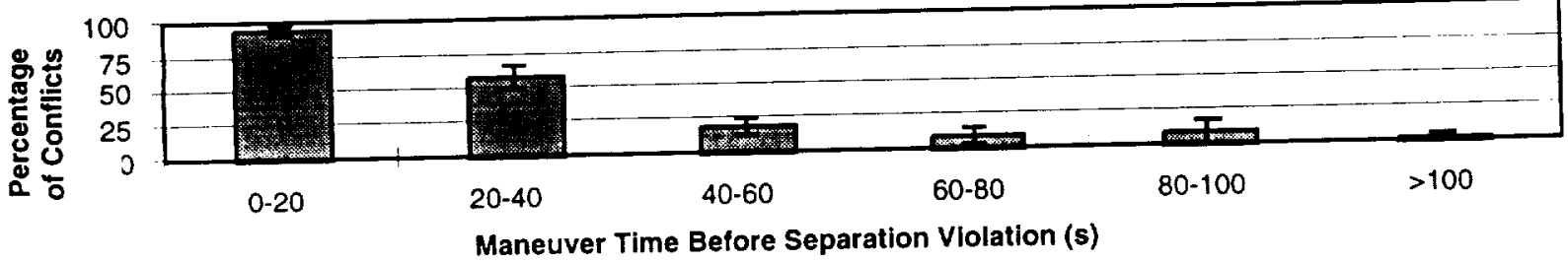

Figure 7: Separation Violation Percentage vs. Maneuver Time 
Subjective Data. The overall rankings for pilot display preference are shown in Figure 8.

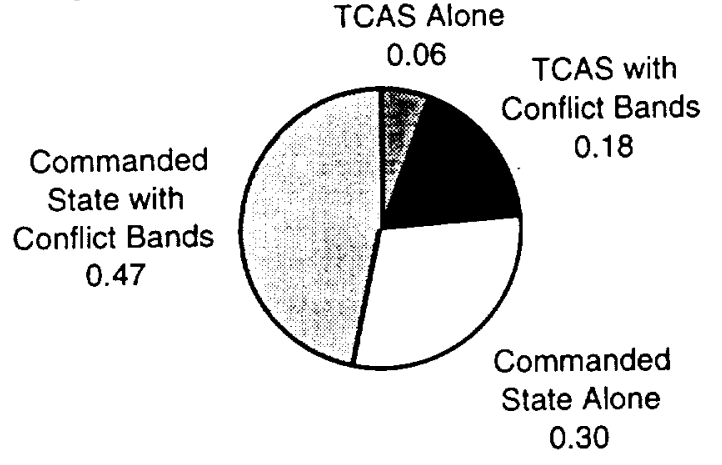

Figure 8: Pilot Display Preference
Pilots preferred all display options over the base TCAS Display by at least a 3:1 margin. They tended to prefer the Commanded State Display with Conflict Bands the most, but if required to make a choice, they would rather have intent information shown directly on the display than incorporated into a conflict probe.

Fifty percent of the pilots found the Commanded State Display to be too cluttered with the conflict bands and $25 \%$ found it too cluttered if alone. Problems with overlaid symbology and intent information shown for non-conflicting aircraft were mentioned.

Discussion. Showing intent information directly on the display and incorporating it into a conflict probe both tended to reduce the number of separation violations and cause pilots to maneuver earlier. As occurred in Experiment 1 , it proved beneficial to maneuver earlier to avoid conflicts, up to about two minutes before the conflict. This result was supported by the similarity in separation violativi. , ercentage between the two minute and long range look-ahead conflict bands. For the conflicts used in this experiment, there seemed to be no additional benefit to alerting to conflicts beyond two minutes.

The latency time between the appearance of a conflict band and when the pilot maneuvered was shorter for the two minute conflict band for both displays. This result suggests that pilots will intentionally defer an avoidance maneuver if they feel the alert is issued too early. Pilots tended to maneuver faster in response to the two minute conflict band when using the Commanded State Display. Commanded state information may reinforce the issuance of an alert, thus reassuring pilots than an avoidance maneuver is appropriate.

A comparison of results between the two experiments suggests that the profile view display is not a vital component. Pilots had no more separation violations on the Intruder Overtake Scenario without the profile view display than they did with the combined plan/profile displays in Experiment 1.

\section{CONCLUSIONS}

Intent information will likely be valuable in the design of an enhanced CDTI. On both experiments, intent information reduced the number of separation violations and caused pilots to maneuver earlier in response to conflicts. Maneuvering early reduced the number of separation violations. However, providing conflict band alerts to conflicts more than two minutes away did not improve performance for the scenarios tested. Providing only information through the next target state appears to be as beneficial as showing the entire FMS-Path. The Commanded State Display performed as well as the FMS-Path Display, would be easier to implement, and was preferred by pilots.

\section{ACKNOWLEDGMENTS}

This study was supported by the NASA Ames Research Center under grant NAG2-716. The authors would also like to thank the pilots who participated in the two experiments.

\section{REFERENCES}

Palmer, E. (1983). Conflict Resolution Maneuvers During Near Miss Encounters with Cockpit Traffic Displays. In Proceedings of the Human Factors Society-27th Amual Meeting (pp. 757-761). Santa Monica, CA: Human Factors Society.

Palmer, E., Jago, S. J., Baty, D. L., \& O'Connor, S. L. (1980). Perception of Horizontal Aircraft Separation on a Cockpit Display of Traffic Information. Human Factors Journal 22(5), 605-620.

Vakil, S. S., Midkiff, A. H., \& Hansman, R. J. (1995). Development and Evaluation of an Electronic Vertical Situation Display. NASA Grant NAG1-1581.

Yang, L. C., \& Hansman, R. J. (1995). Application of the Analytic Hierarchy Process for Making Subjective Comparisons Between Multiple Automation/Display Options. In 6th IFAC/IFIP/IFORS/IEA Symposium on Analysis, Design, and Evaluation of Man-Machine Systems (pp 555-559). Cambridge, MA. 